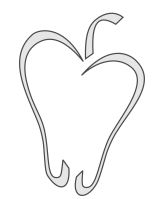

ISSN 1852-4249

\title{
The effect of the lateral interactions on the critical behavior of long straight rigid rods on two-dimensional lattices
}

\author{
P. Longone ${ }^{1}$ D. H. Linares ${ }^{1}$ A. J. Ramirez-Pastor ${ }^{1 *}$ \\ Using Monte Carlo simulations and finite-size scaling analysis, the critical behavior of \\ attractive rigid rods of length $k$ ( $k$-mers) on square lattices has been studied. An ordered \\ state, with the majority of $k$-mers being horizontally or vertically aligned, was found. This \\ ordered phase is separated from the disordered state by a continuous transition occurring at \\ a critical density $\theta_{c}$, which increases linearly with the magnitude of the lateral interactions.
}

\section{Introduction}

The study of systems of hard non-spherical colloidal particles has, for many years, been attracting a great deal of interest and the activity in this field is still growing [1-14]. An early seminal contribution to this subject was made by Onsager [1] with his paper on the isotropic-nematic (I-N) phase transition in liquid crystals. The Onsager's theory predicted that very long and thin rods interacting with only excluded volume interaction can lead to long-range orientational (nematic) order. Thus, at low densities, the molecules are typically far from each other and the resulting state is an isotropic gas. However, at large densities, it is more favorable for the molecules to align spontaneously (there are many more ways of placing nearly aligned rods than randomly oriented ones), and a nematic phase is present at equilibrium.

Interestingly, a number of papers have appeared recently, in which the I-N transition was studied in two dimensions [10-14]. In Ref. [10], the au-

*E-mail: antorami@unsl.edu.ar

1 Departamento de Física, Instituto de Física Aplicada, Universidad Nacional de San Luis-CONICET, Chacabuco 917, D5700BWS San Luis, Argentina. thors gathered strong numerical evidence to suggest that a system of square geometry, with two allowed orientations, shows nematic order at intermediate densities for $k \geq 7$ and provided a qualitative description of a second phase transition (from a nematic order to a non-nematic state) occurring at a density close to 1 . However, the authors were not able to determine the critical quantities (critical point and critical exponents) characterizing the I-N phase transition occurring in the system. This problem was resolved in Refs. [11, 12], where an accurate determination of the critical exponents, along with the behavior of Binder cumulants, showed that the transition from the low-density disordered phase to the intermediate-density ordered phase belongs to the 2D Ising universality class for square lattices and the three-state Potts universality class for honeycomb and triangular lattices. Later, the I-N phase transition was analyzed by combining Monte Carlo (MC) simulations and theoretical analysis $[13,14]$. The study in Refs. $[13,14]$ allowed (1) to obtain $\theta_{c}$ as a function of $k$ for square, triangular and honeycomb lattices, being $\theta_{c}(k) \propto k^{-1}$ (this dependence was already noted in Ref. [10]); and (2) to determine the minimum value of $k\left(k_{\min }\right)$, which allows the formation of a nematic phase on triangular $\left(k_{\min }=7\right)$ and honeycomb $\left(k_{\text {min }}=11\right)$ lattices. 
Papers in Physics, vol. 1, ART. 010005 (2009) / P. Longone et al.

In a recent paper, Fischer and Vink [15] indicated that the transition studied in Refs. [10-14] corresponds to a liquid-gas transition, rather than $\mathrm{I}-\mathrm{N}$. This interpretation is consistent with the 2DIsing critical behavior observed for monodisperse rigid rods on square lattices [11]. This point will be discussed in more detail in Sec. III.

In contrast to the systems studied in Refs. [1014], many rod-like biological polymers are formed by monomers reversibly self-assembling into chains of arbitrary length. Consequently, these systems exhibit a broad equilibrium distribution of filament lengths. A model of self-assembled rigid rods has been recently considered by Tavares et al. [16]. The authors focused on a system composed of monomers with two attractive (sticky) poles that polymerize reversibly into polydisperse chains and, at the same time, undergo a continuous I-N phase transition. The obtained results revealed that nematic ordering enhances bonding. In addition, the average rod length was described quantitatively in both phases, while the location of the ordering transition, which was found to be continuous, was predicted semiquantitatively by the theory.

Beyond the differences between lattice geometry and the characteristics of the rods (self-assembled or not), one fundamental feature is preserved in all the studies mentioned above. This is the assumption that only excluded volume interactions between the rods are considered (except in Ref. [16], where monomers with two attractive bonding sites polymerize into polydisperse rods). Moreover, one often encounters phrases in the literature, such as "This theory [Onsager's theory] shows that repulsive interactions [excluding volume interactions] alone can lead to long-range orientational nematic order, disproving the notion that attractive interactions are a prerequisite" [17], which could be ambiguous with respect to the role that attractive lateral interactions between the rods should play in reinforcing (or not) the nematic order.

In this context, it is of interest and of value to inquire how the existence of lateral interactions between the rods influences the phase transition occurring in the system. The objective of this paper is to provide a thorough analysis in this direction. For this purpose, an exhaustive study of the phase transition occurring in a system of attractive rigid rods deposited on square lattices was performed. The results revealed that $(i)$ the orientational or- der survives in the presence of attractive lateral interactions; (ii) the critical density shifts to higher values as the magnitude of the lateral interactions is increased; and (iii) the continuous transition becomes first order for interaction strength $w>w_{c}$ (in absolute values).

The outline of the paper is as follows. In Sec. II we describe the lattice-gas model and the simulation scheme. In Sec. III we present the MC results. Finally, the general conclusions are given in Sec. IV.

\section{Lattice-gas model and Monte Carlo simulation scheme}

We address the general case of adsorbates assumed to be linear rigid particles containing $k$ identical units ( $k$-mers), with each one occupying a lattice site. Small adsorbates would correspond to the monomer limit $(k=1)$. The distance between $k$ mer units is assumed to be equal to the lattice constant; hence exactly $k$ sites are occupied by a $k$-mer when adsorbed (see Fig. 1). The surface is represented as an array of $M=L \times L$ adsorptive sites in a square lattice arrangement, where $L$ denotes the linear size of the array. In order to describe the system of $N k$-mers adsorbed on $M$ sites at a given temperature $T$, let us introduce the occupation variable $c_{i}$ which can take the values $c_{i}=0$ if the corresponding site is empty and $c_{i}=1$ if the site is occupied. On the other hand, molecules adsorb or desorb as one unit, neglecting any possible dissociation. Under these considerations, the Hamiltonian of the system is given by

$$
H=w \sum_{\langle i, j\rangle} c_{i} c_{j}-N(k-1) w+\epsilon_{o} \sum_{i} c_{i}
$$

where $w$ is the nearest-neighbor (NN) interaction constant which is assumed to be attractive (negative), $\langle i, j\rangle$ represents pairs of NN sites and $\epsilon_{o}$ is the energy of adsorption of one given surface site. The term $N(k-1) w$ is subtracted in eq. (1) since the summation over all the pairs of NN sites overestimates the total energy by including $N(k-1)$ bonds belonging to the $N$ adsorbed $k$-mers. Because the surface was assumed to be homogeneous, the interaction energy between the adsorbed dimer and the atoms of the substrate $\epsilon_{o}$ was neglected for the sake of simplicity. 


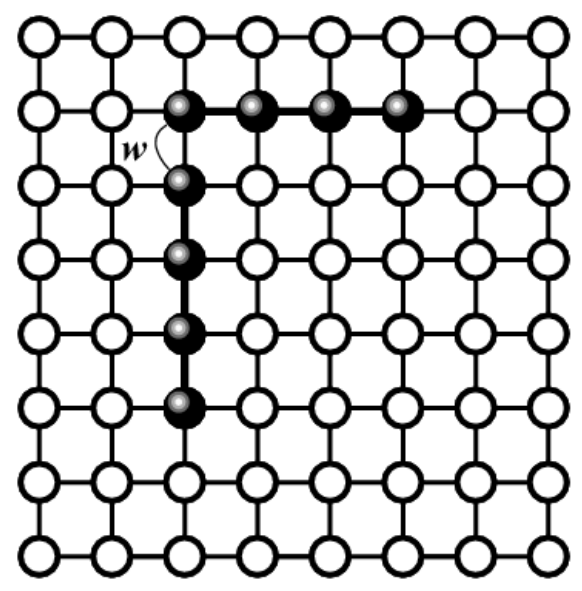

Figure 1: Linear tetramers adsorbed on square lattices. Full and empty circles represent tetramer units and empty sites, respectively.

In order to characterize the phase transition, we use the order parameter defined in Ref. [11], which in this case can be written as

$$
\delta=\frac{\left|n_{h}-n_{v}\right|}{n_{h}+n_{v}}
$$

where $n_{h}\left(n_{v}\right)$ is the number of rods aligned along the horizontal (vertical) direction. When the system is disordered $\left(\theta<\theta_{c}\right)$, all orientations are equivalents and $\delta$ is zero. As the density is increased above $\theta_{c}$, the $k$-mers align along one direction and $\delta$ is different from zero. Thus, $\delta$ appears as a proper order parameter to elucidate the phase transition.

The problem has been studied by grand canonical MC simulations using a typical adsorptiondesorption algorithm. The procedure is as follows. Once the value of the chemical potential $\mu$ is set, a linear $k$-uple of nearest-neighbor sites is chosen at random and an attempt is made to change its occupancy state with probability $W=$ $\min \left\{1, \exp \left(-\Delta H / k_{B} T\right)\right\}$, where $\Delta H=H_{f}-H_{i}$ is the difference between the Hamiltonians of the final and initial states and $k_{B}$ is the Boltzmann constant. In addition, displacement (diffusional relaxation) of adparticles to nearest-neighbor positions, by either jumps along the $k$-mer axis or reptation by rotation around the $k$-mer end, must be allowed in order to reach equilibrium in a reasonable time. A MC step

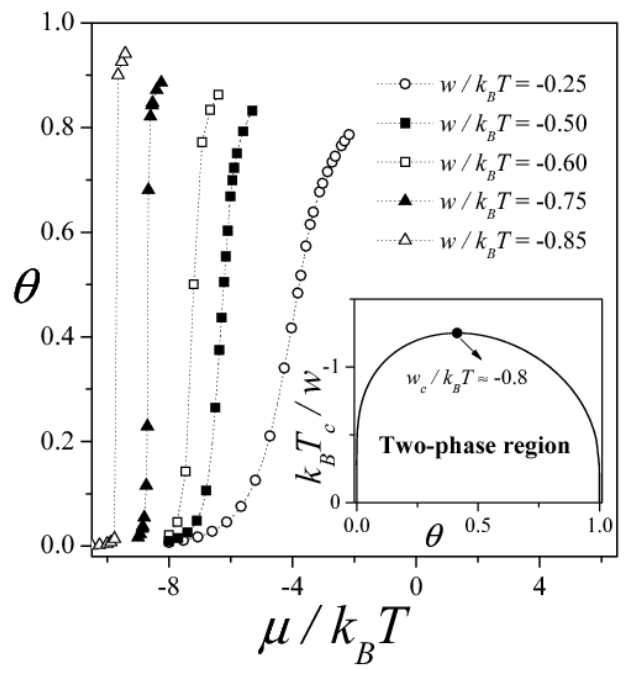

Figure 2: Adsorption isotherms (coverage versus chemical potential) for $k=10, L=100$ different $w / k_{B} T$ 's as indicated. Inset: Adsorption phase diagram of attractive 10-mers on square lattices.

(MCs) is achieved when $M k$-uples of sites have been tested to change its occupancy state. Typically, the equilibrium state can be well reproduced after discarding the first $r^{\prime}=10^{7} \mathrm{MCs}$. Then, the next $r=2 \times 10^{7}$ MCs are used to compute averages.

In our MC simulations, we varied the chemical potential $\mu$ and monitored the density $\theta$ and the order parameter $\delta$, which can be calculated as simple averages. The reduced fourth-order cumulant $U_{L}$ introduced by Binder [18] was calculated as:

$$
U_{L}=1-\frac{\left\langle\delta^{4}\right\rangle}{3\left\langle\delta^{2}\right\rangle^{2}},
$$

where $\langle\cdots\rangle$ means the average over the MC simulation runs. All calculations were carried out using the BACO parallel cluster (composed by $60 \mathrm{PCs}$ each with a $3.0 \mathrm{GHz}$ Pentium-4 processor and 90 PCs each with a $2.4 \mathrm{GHz}$ Core 2 Quad processor) located at Instituto de Física Aplicada, Universidad Nacional de San Luis-CONICET, San Luis, Argentina.

\section{Results}

The calculations were developed for linear 10-mers $(k=10)$. With this value of $k$ and for non- 
Papers in Physics, vol. 1, ART. 010005 (2009) / P. Longone et al.

interacting rods, it is expected the existence of a nematic phase at intermediate densities [10]. The surface was represented as an array of adsorptive sites in a square lattice arrangement with conventional periodic boundary conditions. The effect of finite size was investigated by examining lattices with $L=50,100,150,200$.

In order to understand the basic phenomenology, we consider, in the first place, the behavior of the adsorption isotherms in presence of attractive lateral interactions between the $k$-mers.

Fig. 2 shows typical adsorption isotherms (coverage versus $\mu / k_{B} T$ ) for linear 10-mers with different values of the lateral interaction (the solid circles represent the Langmuir case, $\left.w / k_{B} T=0\right)$.

The isotherms shift to lower values of chemical potential, and their slopes increase as the ratio $w / k_{B} T$ increases (in absolute value). For interaction strength above the critical value $\left(w>w_{c}\right.$, in absolute values) the system undergoes a firstorder phase transition, which is observed in the clear discontinuity in the adsorption isotherms ${ }^{1}$. In the case studied, this critical value is approximately $w_{c} / k_{B} T \approx-0.80$ (or $k_{B} T_{c} / w \approx-1.25$ ). The behavior of the adsorption isotherms also allows us to calculate the phase diagram of the adsorbed monolayer in "temperature-coverage" coordinates. In fact, once obtained the real value of the chemical potential (or critical chemical potential $\mu_{c}$ ) in the two-phase region, the corresponding critical densities can be easily calculated. By repeating this procedure for different temperatures ranging between 0 and $T_{c}$, the coexistence curve can be built [20]. A typical phase diagram, obtained in this case for attractive 10-mers, is shown in the inset of Fig. 2.

On the basis of the study in Fig. 2, our next objective is to obtain evidence for the existence of nematic order in the range $-0.80 \leq w / k_{B} T<0$ of attractive interactions. For this purpose, the behavior of the order parameter $\delta$ as a function of coverage was analyzed for $k=10, L=100$ and dif-

\footnotetext{
${ }^{1}$ In this situation, which has been observed experimentally in numerous systems, the only phase which one expects is a lattice-gas phase at low coverage, separated by a two-phase coexistence region from a "lattice-fluid" phase at higher coverage. This condensation of a two-dimensional gas to a two-dimensional liquid is similar to that of a lattice-gas of attractive monomers. However, the symmetry particlevacancy (valid for monoatomic particles) is broken for $k$ mers and the isotherms are asymmetric with respect to $\theta=0.5$.
}

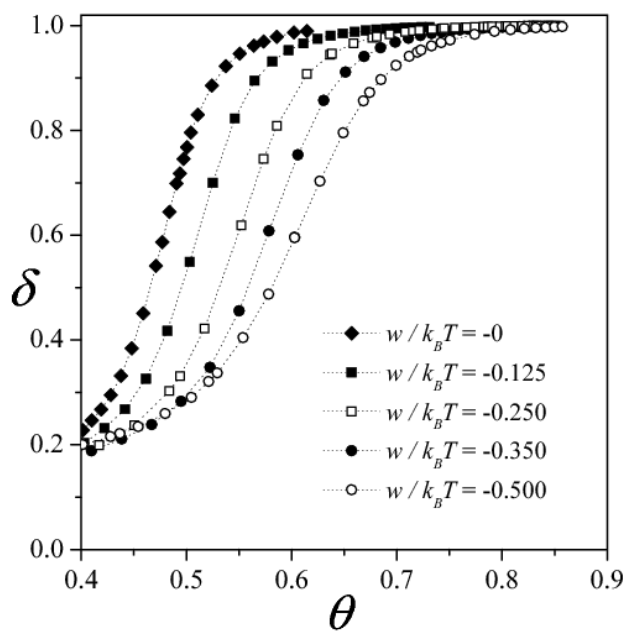

Figure 3: Surface coverage dependence of the nematic order parameter for $k=10, L=100$ different $w / k_{B} T$ 's as indicated.

ferent values of the lateral interaction. The results are shown in Fig. 3, revealing that $(i)$ the orientational order survives in the presence of attractive lateral interactions and $(i i)$ the critical density shifts to higher values as the magnitude of the lateral interactions is increased.

In order to corroborate the results obtained in the last figure, we now study the dependence of $\theta_{c}$ on $w / k_{B} T$. In the case of the standard theory of FSS $[18,19]$, when the phase transition is temperature driven, the technique allows for various efficient routes to estimate $T_{c}$ from MC data. One of these methods, which will be used in this case, is from the temperature dependence of $U_{L}(T)$, which is independent of the system size for $T=T_{c}$. In other words, $T_{c}$ is found from the intersection of the curve $U_{L}(T)$ for different values of $L$, since $U_{L}\left(T_{c}\right)=$ const. In our study, we modified the conventional FSS analysis by replacing temperature by density [11]. Under this condition, the critical density has been estimated from the plots of the reduced four-order cumulants $U_{L}(\theta)$ plotted versus $\theta$ for several lattice sizes. As an example, Fig. 4 shows the results for $w / k_{B} T=-0.125$. In this case, the value obtained was $\theta_{c}=0.542(2)$. In the inset, the data are plotted over a wider range of temperatures, exhibiting the typical behavior of the cumulants in the presence of a continuous phase 
Papers in Physics, vol. 1, ART. 010005 (2009) / P. Longone et al.

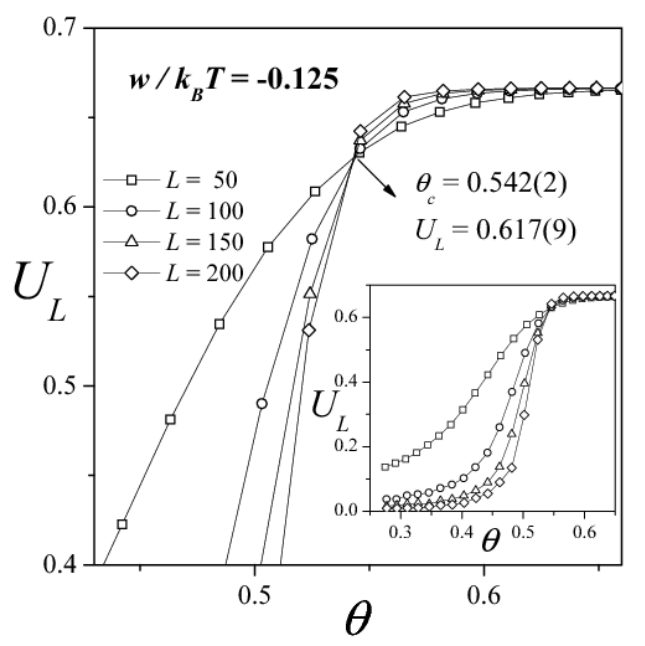

Figure 4: Curves of $U_{L}(\theta)$ vs $\theta$ for $k=10$, $w / k_{B} T=-0.125$ and square lattices of different sizes. From their intersections one obtained $\theta_{c}$. In the inset, the data are plotted over a wider range of densities.

transition.

The procedure of Fig. 4 was repeated for $-0.80 \leq w / k_{B} T<0$, showing that the values of $\theta_{c}$ increase linearly with the magnitude of the lateral couplings (see solid squares in Fig. 5). The critical line (dotted line in the figure) was obtained from the linear fit of the numerical data. As it is possible to observe, the range of coverage at which the transition occurs diminishes as $w / k_{B} T$ is increased (in absolute value). This finding indicates that the presence of attractive lateral interactions between the rods does not favor the formation of nematic order in the adlayer. The phenomenon can be understood from the behavior of the second virial coefficient, which will initially decrease on introducing attractive $w$. This decrease implies that the isotherms shift to lower values of chemical potential, and consequently, the critical point shifts to higher densities.

We did not assume any particular universality class for the transitions analyzed here in order to calculate their critical densities, since the analysis relied on the order parameter cumulant's properties. However, the fixed value of the cumulants, $U^{*}=0.617(9)$, is consistent with the extremely precise transfer matrix calculation of $U^{*}=$

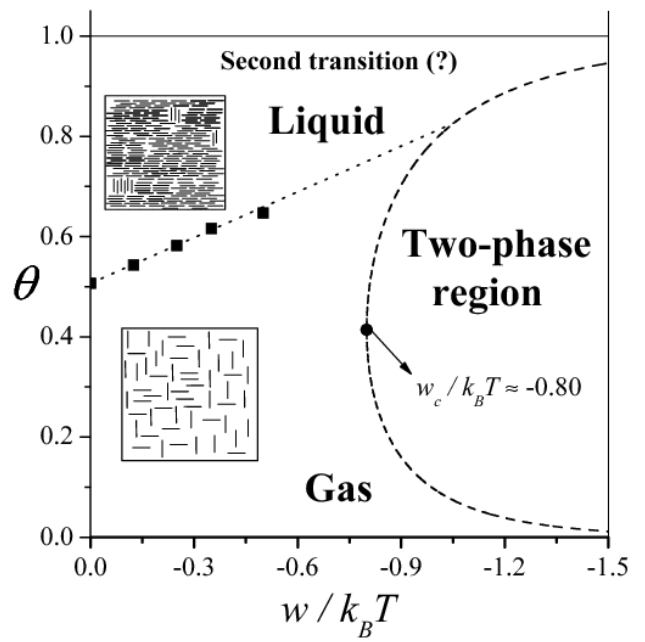

Figure 5: Temperature-coverage phase diagram corresponding to attractive $k$-mers with $k=$ 10. The inset in the upper-left (lower-right) corner shows a typical configuration in the nematic (isotropic) phase.

0.6106901(5) [21] for the 2D Ising model. This finding may be taken as an indication that the phase transition belongs to the 2D Ising universality class.

With respect to the behavior of the system for $w / k_{B} T<-0.80$, the adsorbed layer "jumps" from a low-coverage phase to a high-coverage phase. This effect, which has been discussed in Fig. 2, is represented in Fig. 5 by the dashed coexistence line. The low-coverage phase is an isotropic state, similar to that observed for $w / k_{B} T>-0.80$ and low density (see inset in the lower-right corner of Fig. 5). On the other hand, the high-coverage phase is also an isotropic state, but characterized by the presence of local orientational order (domains of parallel $k$-mers). A typical configuration in this regime is shown in Fig. 6).

Finally, it is worth pointing out that: (1) the behavior of the order parameter in Fig. 3 clearly indicates that the transition from the low-density disordered phase to the intermediate-density ordered phase is an isotropic to nematic phase transition (when all the words have the usual meaning). In this case, the transition under study belongs to the $2 \mathrm{D}$ Ising universality class. It can also be thought of as an unmixing or liquid-gas transition [15]. For this reason we have called gas and liquid to the 
Papers in Physics, vol. 1, ART. 010005 (2009) / P. Longone et al.

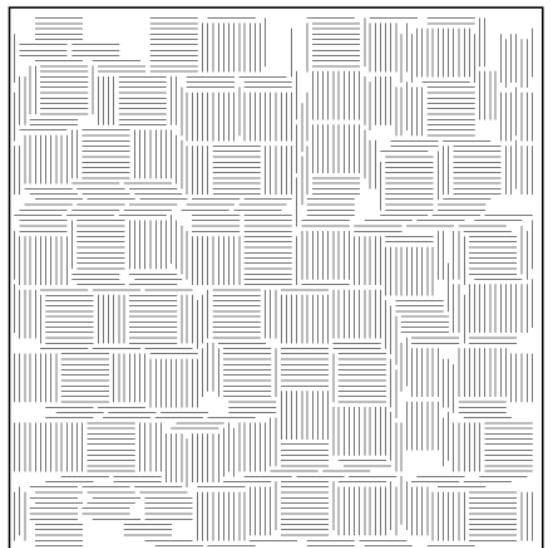

Figure 6: Typical configuration of the adlayer in the high-coverage phase and $w / k_{B} T<-0.80$.

phases reported in Fig. 5; and (2) even though it has not been rigorously proved yet, a second phase transition for non-interacting rods at high densities has been theoretically predicted [10] and numerically confirmed [13]. This result has not been confirmed for the case of attractive rods. An exhaustive study on this subject will be the object of future work.

\section{Conclusions}

We have addressed the critical properties of attractive rigid rods on square lattices with two allowed orientations, and shown the dependence of the critical density on the magnitude of the lateral interactions $w / k_{B} T$. The results were obtained by using MC simulations and FSS theory.

Several conclusions can be drawn from the present work. On the one hand, we found that even though the presence of attractive lateral interactions between the rods does not favor the formation of nematic order in the adlayer, the orientational order survives in a range that goes from $w / k_{B} T=0$ up to $w_{c} / k_{B} T \approx-0.80\left(w_{c} / k_{B} T\right.$ represents the critical value at which occurs a typical transition of condensation in the adlayer). In this region of $w / k_{B} T$, the critical density increases linearly with the magnitude of the lateral couplings. On the other hand, the evaluation of the fixed point value of the cumulants $U^{*}=0.617(9)$ indicates that, as in the case of non-interacting rods, the observed phase transition belongs to the universality class of the two-dimensional Ising model.

With respect to the behavior of the system for $w / k_{B} T<-0.80$, the continuous transition becomes first order. Thus, the adsorbed layer jumps from a low-coverage phase, similar to that observed for $w / k_{B} T>-0.80$ and low density, to an isotropic phase at high coverage, characterized by the presence of local orientational order (domains of parallel $k$-mers)

Future efforts will be directed to (1) extend the study to repulsive lateral interaction between the $k$-mers; (2) obtain the whole phase diagram in the space (temperature-coverage-rod's size); (3) develop an exhaustive study on critical exponents and universality and (4) characterize the second phase transition from a nematic order to a non-nematic state occurring at high density.

Acknowledgements - This work was supported in part by CONICET (Argentina) under project number PIP 112-200801-01332; Universidad Nacional de San Luis (Argentina) under project 322000 and the National Agency of Scientific and Technological Promotion (Argentina) under project 33328 PICT 2005.

[1] L Onsager, The effects of shape on the interaction of colloidal particles, Ann. N. Y. Acad. Sci. 51, 627 (1949).

[2] P J Flory, Thermodynamics of high polymer solutions, J. Chem. Phys. 10, 51 (1942); P J Flory, Principles of Polymers Chemistry, Cornell University Press, Ithaca, NY (1953).

[3] M L Huggins, Some properties of solutions of long-chain compounds, J. Phys. Chem. 46, 151 (1942); M L Huggins, Thermodynamic properties of solutions of long-chain compounds, Ann. N. Y. Acad. Sci. 43, 1 (1942); M L Huggins, Theory of solutions of high polymers, J. Am. Chem. Soc. 64, 1712 (1942).

[4] J P Straley, Liquid crystals in two dimensions, Phys. Rev. A 4, 675 (1971). 
Papers in Physics, vol. 1, ART. 010005 (2009) / P. Longone et al.

[5] J Vieillard-Baron, Phase transitions of the classical hard-ellipse system, J. Chem. Phys. 56, 4729 (1972).

[6] D Frenkel, R Eppenga, Evidence for algebraic orientational order in a two-dimensional hardcore nematic, Phys. Rev. A 31, 1776 (1985).

[7] K J Strandburg, Two-dimensional melting, Rev. Mod. Phys. 60, 161 (1988).

[8] A J Phares, F J Wunderlich, Thermodynamics and molecular freedom of dimers on plane triangular lattices, J. Math. Phys. 27, 1099 (1986).

[9] A J Phares, F J Wunderlich, J D Curley, D W Grumbine Jr, Structural ordering of interacting dimers on a square lattice, J. Phys. A: Math. Gen. 26, 6847 (1993).

[10] A Ghosh, D Dhar, On the orientational ordering of long rods on a lattice, Eur. Phys. Lett. 78, 20003 (2007).

[11] D A Matoz-Fernandez, D H Linares, A J Ramirez-Pastor, Determination of the critical exponents for the isotropic-nematic phase transition in a system of long rods on twodimensional lattices: Universality of the transition, Europhys. Lett. 82, 50007 (2008).

[12] D A Matoz-Fernandez, D H Linares, A J Ramirez-Pastor, Critical behavior of long linear $k$-mers on honeycomb lattices, Physica A 387, 6513 (2008).

[13] D H Linares, F Romá, A J Ramirez-Pastor, Entropy-driven phase transition in a system of long rods on a square lattice, J. Stat. Mech. P03013 (2008).
[14] D A Matoz-Fernandez, D H Linares, A J Ramirez-Pastor, Critical behavior of long straight rigid rods on two-dimensional lattices: Theory and Monte Carlo simulations, J. Chem. Phys. 128, 214902 (2008).

[15] T Fischer, R L C Vink, Restricted orientation "liquid crystal" in two dimensions: Isotropicnematic transition or liquid-gas one(?), Europhys. Lett. 85, 56003 (2009).

[16] J M Tavares, B Holder, M M Telo da Gama, Structure and phase diagram of self-assembled rigid rods: Equilibrium polydispersity and nematic ordering in two dimensions, Phys. Rev. E 79, 021505 (2009).

[17] H H Wensink, Columnar versus smectic order in systems of charged colloidal rods, J. Chem. Phys. 126, 194901 (2007).

[18] K Binder, Applications of the Monte Carlo Method in Statistical Physics. Topics in current Physics, Springer, Berlin (1984).

[19] V Privman, Finite Size Scaling and Numerical Simulation of Statistical Systems, World Scientific, Singapore (1990).

[20] T L Hill, An Introduction to Statistical Thermodynamics, Addison Wesley Publishing Company, Reading, MA (1960).

[21] G Kamieniarz, H W J Blöte, Universal ratio of magnetization moments in two-dimensional Ising models, J. Phys. A: Math. Gen. 26, 201 (1993). 